there are effective treatments which can improve the prognosis for a child. The signs are that in approximately ten years we will have reached a point where a minimal service should be available nationally. Looking at medical manpower projections, it would be unrealistic to call for a set of new increased norms. The solution will have to lie in ensuring that every district at least meets the 1983 norms, but equally that other Health Service professionals such as community psychiatric nurses and child psychotherapists are available to participate in this work. Serious consideration should be given by health districts to providing other forms of workers, such as family and behavioural therapists, who can assist consultants in their work.

\section{References}

Royal College of Psychiatrists (1983) Providing a district service for child and adolescent psychiatry: medical manpower priority. Bulletin of the Royal College of Psychiatrists, 7, 94-97.

Wressell, S. E., Kaplan, C. A. \& Kolvin, I. (1989) Performance indicators and child sexual abuse. Psychiatric Bulletin, 13, 599-601.

Approved by Executive and Finance Committee.

November 1990

\title{
Research by trainees
}

\section{A report by the Working Party of the Collegiate Trainees' Committee}

\section{Introduction}

The vexed question of research by psychiatric trainees has continued to plague us. In 1980 the Royal College of Psychiatrists devoted an entire session of the autumn quarterly meeting to psychiatric research, the papers later published in the Bulletin in 1981.

Professor Goldberg (1981) described a new course - Encouraging Psychiatric Trainees to Undertake Research. Hollyman \& Abou Saleh (1985) reported the findings of a survey of Southern Division trainees performed by Waters in 1980. It was apparent that by 1985 several centres nationally had begun local courses in research methodology. However, in 1986 reports began reaching the Collegiate Trainees' Committee from Divisional Trainees' Days of concern being voiced by trainees themselves. Crauford et al (1986) highlighted this area in their report of the North West Trainees' Day.

Two main problem areas were noted. Trainees felt under pressure to 'do research' to enhance career prospects when they felt the time to learn or develop clinical skills was limited already, a view expressed particularly by post MRCPsych registrars, compet- ing for SR posts, given the current bottleneck at this level. Further, having decided to embark on research, multiple practical problems in carrying out the research were being encountered and training, advice, and practical support were hard to obtain.

A working party was convened to examine some of the issues and try to discover the current situation.

\section{Method}

Information from the trainees' days was noted. A questionnaire was distributed to 890 trainees in five divisions of the College. The three main sections covered demographic details, research training, experience and supervision, and finally an open narrative section on how the situation might be improved.

\section{Results}

There was a $33 \%$ response rate with 296 usable questionnaires returned. The demographic details showed an equal response rate from all divisions sampled. The respondents posts held are skewed 
towards senior trainees, $81 \%$ having passed MRCPsych with normal age and equal sex distribution; $54 \%$ of respondents worked in university linked teaching hospitals, $16.5 \%$ in peripheral psychiatric units, $14.5 \%$ in district general hospital units and $11.1 \%$ in the Special Health Authority. Part-time trainees accounted for $12.5 \%$ of the sample.

\section{Time allocated}

The majority of trainees reported time allocated for research varying between one to two sessions per week $(47.9 \%)$ and full-time $(7 \%)$. However, $14.8 \%$ reported that no time was allocated to research and none available with an additional $17.2 \%$ fitting in research time although none was allocated. An all too common comment was also that although time was allocated pressure of clinical work often precluded its use.

\section{Encouragement/discouragement}

Alarmingly, $16.2 \%$ reported active discouragement in pursuing research. Of this former group eight own consultants, two clinical tutors, five local consultants and fifteen non-medical personnel and peers were discouraging in their approach. A further unfortunate $17.6 \%$ of respondents reported no encouragement in carrying out their research. The presence of total apathy in peers and colleagues was identified in $32 \%$.

\section{Training}

Twenty-two per cent believed that research training should begin at medical school or earlier, encouraging the development of an enquiring, research orientated mind from an early age; $69 \%$ indicated that the SHO/registrar level was probably the most appropriate level. Less than $10 \%$ felt that research training should be left till the post-membership stage.

In response to the availability and adequacy of research training, $59.4 \%$ stated research training was available. Of those, $63 \%$ said a local course was available and two-thirds had attended. However, $40.5 \%$ were unaware of any available research training locally or nationally.

Only $23.9 \%$ rated their research training as adequate for their needs. Those in the Midland Division had only $8 \%$ of trainees satisfied whereas $34.6 \%$ of those in the North East were satisfied.

When analysed for type of hospital, the Special Health Authority trainees were most satisfied with research training $(42.4 \%)$ but only $13.9 \%$ of those in DGH units felt they had adequate research training.

\section{Supervision}

The majority of trainees $(67 \%)$ were unhappy with their supervision. However, this ranged from $36 \%$ of those in the Special Health Authority to $87.7 \%$ of those working in peripheral psychiatric units, and $86 \%$ of those in district general units.

Across the country satisfaction with supervision did not vary greatly; Southern trainees $(38 \%)$ were most satisfied, South West trainees $(20 \%)$ least.

Supervision and support was sought generally from senior colleagues with either sound research experience or interest in a particular clinical topic. Many, however, reported supervision was still difficult to obtain. In this respect the research forums were seen as fulfilling a valuable role provided they were attended by senior staff in addition to trainees.

Research forums were available for $77 \%$ of the respondents; $60 \%$ of these were held in the respondent's own hospital. Of research forums, hospital based, district or regional, $60 \%$ were held more frequently than quarterly.

\section{Research experience}

Of those who responded, $95 \%$ had been actively involved in research. Further, $83.4 \%$ of these felt research activity should begin during or before registrar appointments.

Of the respondents engaged in research, $86 \%$ had entered the research project at or before the protocol stage.

The stage of research reached was variable, but satisfaction with both training and supervision correlated with progress.

\section{Unfinished projects - failed or stuck}

Many trainees reported at what stages of research a project had become bogged down. This was most commonly 'writing up' (22\%), although 'protocol writing' and 'data analysis' were identified as equally problematic areas (15\%).

The only factors found to correlate with the stage at which a project stuck was satisfaction with research training and supervision. The least satisfied tending to fail at earlier stages.

Many felt inadequate supervision led to poorly designed and sometimes impractical or overambitious studies. Some complained of discouragement and negative comments from supervisors or tutors, without any constructive criticism. Often, ideas were in plentiful supply but the help required to develop these was lacking.

Other practical problems included difficulty gaining access to statistical and computing advice and facilities, lack of funds, lost data and moving to a different job. Some studies faltered due to difficulties 
recruiting subjects, or the poor cooperation of colleagues. Commonly projects were unfinished when findings were negative. Unfortunately, this can be a disincentive to publish. Trainees pointed out that unless genuinely interested in the project, many lacked the determination to pursue it, when faced with these obstacles. Research embarked upon for reasons other than genuine interest seemed therefore more likely to fail.

\section{Full-time research posts}

A surprisingly large percentage $(61 \%)$ of respondents expressed an interest in full-time research posts. For most, the attraction was the prospect of carrying out worthwhile research and producing important, publishable results. Others, however, clearly perceived these posts as valuable in terms of career advancement.

Many made it clear that their preference for clinical work would prevent them from considering fulltime research. Some had thought about it but had abandoned the idea because they knew of no appropriate posts, in the right place at the right time. Family commitments prevented some from moving to obtain a post. Research posts are often financially less rewarding with less job security. This discouraged some. Older trainees, keen to make the consultant grade, perceived that full-time research posts could impede their progress.

\section{The research option of the MRCPsych}

Very few trainees take up the research option of the MRCPsych examination. Although some are simply unaware of its existence (18\%), most $(80 \%)$ do not even consider it. The main priority of pre-membership trainees is naturally to pass the exam. Taking up the research option is perceived as reducing the chances of success. Trainees prefer to concentrate on revising for the exam, and acquiring a broad knowledge of clinical psychiatry. Already pressed for time, exemption from the essay paper is not seen as sufficient incentive to put in the extra work involved in the research option. A problem which may be overcome if the College made it a condition of taking up the research option, that research sessions had been allocated to the trainee. As few trainees or even tutors know what it entails or the standard expected, most believe it is safer, easier and more predictable to sit the standard exam. Many trainees are not interested in research at the pre-membership stage and few have suitable projects for submission.

Some expressed their reluctance to carry out research which they believed could be submitted for the exam but not for a higher degree or publication. The latter two options were perceived as being more meaningful outcomes for research.

\section{Why do research?}

Although reservations were expressed about all trainees having to do research, most perceived it as potentially a positive, creative experience. The commonest reason given for doing research was that it developed an open enquiring mind and a critical approach which challenged both published research and accepted clinical practice. It was felt that this in turn promoted improved standards of clinical practice.

Contributing to the body of psychiatric knowledge was viewed as important. Doing research also encourages in-depth thinking and develops expertise in specific areas. A small number commented that research was stimulating and enjoyable, adding an extra fascinating dimension to their work.

Experience of research as a trainee encourages the view that research is part of life-long clinical practice, as well as developing research skills. This can lead to consultants pursuing research throughout their careers. There is also the advantage that trainees who have sound experience of research will make better research supervisors themselves.

Some pointed out that it was only through being forced to do research that they realised that they actually enjoyed it and saw the positive aspects of research. They argued that this was a good reason for all trainees being exposed to research opportunities.

A significant minority of respondents resented the current pressure on trainees to do research. They believed that excessive emphasis was placed on it at the expense of clinical training. Research and publications are often perceived as a means to career advancement. Many thought this devalued research. Some commented that research for the wrong reasons was a waste of time in particular for those without the interest or ability to pursue high quality research.

\section{How could the College motivate trainees to do research?}

Trainees pointed out that most were already well motivated, in particular because of the current SR job market. However many have difficulties pursuing research because of the lack of support and encouragement. It was generally accepted that if a well organised research infrastructure existed and trainees had adequate time allocated for research, there would be little need for additional motivation by the College.

Many felt that already much poor quality research was carried out, motivated by the necessity to enhance one's CV, and that the College should accept that not everyone can be motivated to produce worthwhile research. 
However, suggestions for motivating trainees to do research included, making it a mandatory requirement for the MRCPsych or Higher Professional Training Prizes, presentations at College meetings or a Research Diploma could also provide incentives. If research could contribute credits towards the MRCPsych, this may encourage trainees.

\section{The Research Unit}

Few had any idea of the purpose of the Research Unit. However, it was clear that trainees believed it should provide the central training and back up facilities needed, to help trainees to do research.

\section{Discussion}

The low response rate (33\%) and undoubtedly skewed population towards senior and university linked trainees with $95 \%$ research activity must surely be a self-selected and motivated group not representative of the population of trainees in the five divisions surveyed. However, if even this motivated group reported major difficulties in attempting research, the views of almost 300 trainees must be of some value.

It was reassuring to note most respondents were able to set aside research time but worrying that so many commented on the practical difficulties in doing this.

Research methodology training has now been available nationally since 1987 and longer in some local areas; that $40 \%$ of respondents claimed no availability of training is concerning and must question the effectiveness of the publicity.

While one might have expected that teaching units conferred research advantages, this was not universally borne out with only research activity by local or own consultants in these units correlating with availability of training and supervision. Respondents were as active in research in other types of units but reported major difficulties in obtaining appropriate support and practical help.

As in our anecdotal reports, protocol writing, data analysis and writing up were the most common reasons for a project to get bogged down. However project failure and abandonment in addition also involved problems with funding, pilot study and knowing how to handle negative data. It would seem that with constructive advice many of these projects could have been salvaged preserving the morale of the researcher.

Perhaps it is now time for more co-ordinated action based on the observations of trainees.

Many trainees commented positively about research, but expressed their frustrations about the obstacles faced. They made realistic and constructive suggestions on how the College could help to overcome these on both a local and central level. Although a few were grateful for having been forced into doing research, as they had discovered through this that they had a flair for it, many resented this pressure. Research for CV purposes and career advancement was viewed with distaste. Many felt that their strengths lay in the clinical field and rather than persuading them to do research, the College should be concentrating on helping those with genuine interest. It is difficult to see a solution to this dilemma. There is no doubt that research can be a very valuable experience which enhances one's clinical practice. However, it seems iniquitous that people who chose not to do research are penalised as they may be very able clinicians.

It would appear that little has changed since Szmukler presented his observations in 1981 and Hollyman \& Abou Saleh in 1985.

\section{Recommendations}

(1) JCHPT should continue to apply pressure on consultants to ensure that the two research sessions which are allocated to SRs are available in 'real' terms.

(2) However, it is recognised that the Research Committee organises National Research Methodology courses on a regular basis for both trainees and research supervisors. JCHPT and the Central Approval Panel should actively encourage the running of research seminars on a local level, which are attended by experienced researchers and supervisors as well as trainees.

(3) The College should develop a central facility devoted purely to research. The functions of such a facility would be manifold and should be available to trainees from all schemes, facilitated by modern communication technology.

(a) Training: research, statistics (should also be available for consultants)

(b) Central reference point: Register of experienced researchers/experts in specific fields

Register of research posts; including six month posts on rotations

Instigation and co-ordination of multicentre research suitable for trainees

Advice on sources of funding and grant applications

(c) Specific problem solving:

Practical advice, e.g. funding, ethics, data handling

High quality research supervision/specific problem solving advice

Access to computerised literature searching

(d) Research Promotion:

Fundraising, Research Fellowships, Prizes. 
It is imperative, however, that the benefits of such a facility should be available to all trainees and not only centrally based units by virtue of personal contact or proximity.

(4) The Examinations Department should consider making a condition of taking up the research option of the MRCPsych, that weekly research sessions are allocated.

(5) The College should be encouraging the appointment at senior registrar level of those who are suitable to subsequently become appointed as consultants. Although assessment of research experience may be a simple way to decide appointments, the clinical and organisational skills of the applicant should carry more weight with the appointing committee.

S. J. Johnston, Convenor J. SMITH, Co-author

\section{Acknowledgements}

Thanks to working party members who helped to draft the questionnaire over the duration: G. Bell, C. Bools, F. Browne, R. Cawthra, J. Cooney, I. Medley, R. Polley, C. Sullivan and P. White. Thanks also to C. Freeman for his comments on the draft.

Further acknowledgements must go to: The Research Commitee who supported our application for postage funding; Jean Wales for her encouragement and practical advice; Drs Nutt and Glue of the Reckitt and Colman Psychopharmacology Unit, Bristol Royal Infirmary for advice and support with the data processing and June Johnstone our very patient secretary without whom we would still be struggling.

Approved by Executive and Finance Committee, September 1990. Circulated for wider debate within the College.

\section{References and further reading}

BlaCK, D. (1981) The outside view. Bulletin of the Royal College of Psychiatrists, 5, 38-39.

Crauford, D., Davidson, I. \& Simpson, M. (1986) North West Division Trainees Day. Bulletin of the Royal College of Psychiatrists, 10, 16-17.

GOLDBERG, D. (1981) Encouraging psychiatric trainees to do research - a preliminary account of a new course. Bulletin of the Royal College of Psychiatrists, 5, 87-88.

Hollyman, J. A.\& ABOU-SAlEH, M. T. (1985) Trainees and research. Bulletin of the Royal College of Psychiatrists, 9, 203-204.

RAWNSLEY, K. (1981) The inside view. Bulletin of the Royal College of Psychiatrists, 5, 49-50.

Sims, A. C. P. (1981) Research and the Royal College of Psychiatrists. Bulletin of the Royal College of Psychiatrists, 5, 45-46.

SzMUKLER, G. (1981) Tinker, trainer, Savoir-faire - the trainee and research. Bulletin of the Royal College of Psychiatrists, 5, 47-48.

WOOD, G. M. \& CATFORD, J. C. (1986) Research post MRCP. Journal of Royal College of Physicians, 20, 89-94.

Copies of the seven Figures giving demographic details, etc, are available from Jean Wales at the College.

\section{Report of the Collegiate Trainees' Committee Working Party on training of junior psychiatrists with respect to violent incidents}

In September 1989, a working party of the Collegiate Trainees' Committee (CTC) was set up to examine issues in relation to the involvement of trainee psychiatrists in dealing with actual or threatened violence.

\section{Background}

The CTC had become concerned about the issue of the personal safety of junior psychiatrists as a result of incidents known to members of the Committee when trainees had been assaulted during the course of their work or had been pestered or threatened by patients when off duty. The aim of the working party was to explore the problem and, as quickly as possible, to develop some recommendations which might be readily implemented by the College and which would have a significant impact in improving the safety and training of junior psychiatrists. 